Magneto-optical Imaging of Transport Curreht Belsities in Superconductors*

G.W. Crabtree, ${ }^{1}$ U. Welp, ${ }^{1}$ D.O.Gunter, ${ }^{1}$ W. Zhong, ${ }^{2}$ U. Balachandran, ${ }^{2}$ P. Haldar, ${ }^{3}$

R.S. Sokolowski, ${ }^{3}$ V.K. Vlasko-Vlasov, ${ }^{4}$ V.I. Nikitenko ${ }^{4}$

1Materials Science Division
2Energy Technology Division

Argonne National Laboratory, Argonne, Illinois 60439

IIntermagnetics General Corporation, Latham, NY. 12110

${ }^{4}$ Institute for Solid State Physics, 142432 Chernogolovka, Moscow District, Russia

The submitled manuscript has been authored by a
contractor of the U.S. Government under contract
Na. W-31-10G-ENG-38. Acoordingly, the U.S.
Government retains a nonexclusive, royally-free
hoense to publish or reproduce the published form
of this contribution, or allow others to do so, for
U.S. Government purposes.

Proceedings of the 8th International Symposium on Superconductivity, October 30-November 2, 1995, Hamamatsu, Japan, Springer-Verlag, Tokyo

\title{
DISCLAIMER
}

\begin{abstract}
This report was prepared as an account of work sponsored by an agency of the United States Government. Neither the United States Government nor any agency thereof, nor any of their employees, makes any warranty, express or implied, or assumes any legal liability or responsibility for the accuracy, completeness, or usefulness of any information, apparatus, product, or process disclosed, or represents that its use would not infringe privately owned rights. Reference herein to any specific commercial product, process, or service by trade name, trademark, manufacturer, or otherwise does not necessarily constitute or imply its endorsement, recommendation, or favoring by the United States Government or any agency thereof. The views and opinions of authors expressed herein do not necessarily state or reflect those of the United States Government or any agency thereof.
\end{abstract}

\footnotetext{
*Work supported by the U.S. Department of Energy, BES-Materials Sciences (UW, GWC) and Energy Efficiency and Renewable Energy, as part of a program to develop electric power technology (WZ, UB) under contract \#W-31109-ENG-38 and the National Science Foundation-Office of Science and Technology Centers for Superconductivity under contract \#DMR91-20000 (DOG), by the New Independent States-Industrial Partnership Program (VKV), and by the International Science Foundation under grants \#RF1000 and \#RF1300 (VKV, VIN).
}

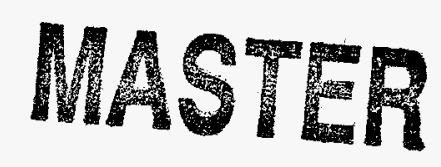


Procecdings of the $8^{\text {th }}$ International Symposium on Superconductivity

October 30 - November 2, 1995. Hamamatsu. Japan

Springer-Verlag Tokyo

\section{Magneto-optical Imaging of Transport Current Densities in Superconductors}

G. W. Crabtree ${ }^{1}$, U. Welp 1 , D. O. Gunter ${ }^{1}$. W. Zhong2, U. Balachandran 2 ,

P. Haldar ${ }^{3}$. R. S. Sokolowski ${ }^{3}$, V. K. Vlasko-Vlasov 1.4 , and V. I. Nikitenko 4

${ }^{1}$ Materials Science Division. Argonne National Laboratory. Argonne, IL 60439 USA

2Energy Technology Division. Argonne National Laboratory. Argonne, IL 60439 USA

Intermagnetics General Corporation, PO Box 461, Latham. NY 12110-0461 USA

4 Institute for Solid State Physics, 142432 Chernogolovka, Moscow District, Russia

\section{ABSTRACT}

A technique for imaging the path and magnitude of the transport current density flowing in superconductors is described. Results are given for a 37-filament BSCCO 2223 powder-intube wire, showing a highly inhomogeneous current path within the filaments.

KEYWORDS: magneto-optical imaging, transport current, microstructure, multifilament wire

\section{INTRODUCTION}

Direct imaging of the paths of transport currents in superconductors creates many new possibilities for exploring the basic features of vortex pinning mechanisms and for improving the performance of superconducting materials. Prior to the application of magneto-optical imaging techniques to. this problem, information on the transport current path was available only on a large scale, determined by the experimental space required for traditional I-V measurements. Typically, voltage and current leads can be placed on samples at intervals of several tens to hundreds of microns, allowing a sampling of the current distribution on this scale. However, the current distribution is determined to a large extent by the microstructure of the underlying material, which typically changes on the scale of microns. Thus, detailed information on the effect of a particular microstructure on the current distribution is difficult to obtain. Furthermore, to determine the critical current as a function of position, it is necessary to cut the sample into slices which are measured individually, thereby avoiding current sharing with neighboring regions which may have a different critical current. While such slicing techniques measure the average critical current over the slice, they do not provide a picture of the actual current distribution as it would flow in the full sample, including the role of connectivity and weak links in guiding the current.

In principle, magneto-optical techniques alleviate the limitations of spatial scale and slicing that are inherent in traditional I-V techniques. Magneto-optical images [1,2] with a resolution in the 1-5 micron range can be obtained with careful optimization of the optical system, and an area with dimensions of tens to hundreds of microns can be studied in a single image. The central problem in making such studies is the recovery of the current information from the field images obtained in the experiment. The approaches to this problem fall into two general categories: (i) application of an external magnetic field with zero applied current, with analysis of the field penetration via the Bean critical state model (shielding mode) [3-7], and (ii) the application of a transport current in zero applied field. with analysis of the self-field patterns to infer the actual current distribution (transport mode) $[4,5.8]$. Applied field techniques typically give the critical current as a function of position, but in practice reveal little information about the actual paths and magnitude of the transport current which is regulated by weak links. Applied current techniques have the advantage of showing the performance of the superconductor under close-to-operating conditions, but they suffer from the notoriously difficult problem of inferring accurate current information from the self-field distribution.

In this paper, we describe an applied current technique for imaging the current flow in superconductors, paying close attention to the problem of inverting the self-field patterns to recover the current distribution. We present a new analysis technique, applicable to long wires, which provides quantitative error limits on the inferred values of the current densities. We apply the technique to commercial multifilament conductors and 
demonstrate a high spatial correlation between microstructure and current density on the micron scale.

\section{INVERSION PROCEDURE}

The simplest inversion procedure for inferring the current distribution from its self-field is based on Ampere's law, $\nabla \times B=(4 \pi / c) J$, where $B$ is the magnetic field and $J$ is the current density. If $J$ is confined to the $x y$ plane. Ampere's law simplifies to

$$
\frac{\partial \mathrm{B}_{\mathrm{z}}}{\partial \mathrm{y}}-\frac{\partial \mathrm{B}_{\mathrm{y}}}{\partial \mathrm{z}}=\frac{4 \pi}{\mathrm{c}} \mathrm{J}_{\mathrm{x}} \quad \frac{\partial \mathrm{B}_{\mathrm{x}}}{\partial \mathrm{z}}-\frac{\partial \mathrm{B}_{\mathrm{z}}}{\partial \mathrm{x}}=\frac{4 \pi}{\mathrm{c}} \mathrm{J}_{\mathrm{y}}
$$

Thus, the current density $J$ is completely specified by measuring the field gradients in Eq. (1). provided the gradients are measured inside the sample where the current is flowing. Magneto-optical experiments face two shortcomings in this regard: they are sensitive only to $\mathrm{B}_{\mathrm{z}}$. not $\mathrm{B}_{\mathrm{x}}$ or $\mathrm{B}_{\mathrm{y}}$. and the measurements are made outside the sample where $J$ is zero. The second shortcoming is not serious for measuring the gradients of $B_{z}$. because the boundary condition on $B$ requires that its normal component be continuous across the boundary. Thus if the boundary is perpendicular to the $z$-axis, the gradients of $B_{z}$ in Eq. (1) are continuous across the boundary and are correctly measured outside the sample in the usual magnetooptical geometry. However, there is no such boundary condition for $\mathrm{B}_{\mathbf{x}}$ or $\mathrm{B}_{\mathbf{y}}$, and in fact their gradients with $z$ change sharply at the surface. The absence of information on $\partial B_{x} / \partial z$ and $\partial \mathrm{B}_{\mathrm{y}} / \partial \mathrm{z}$ (hereafter called the "parallel gradients of $\mathrm{B}$ ") inside the sample in the magnetooptical experiment is a severe limitation on determining $\mathbf{J}$. It is tempting to assume that their contribution is minor compared to that of the perpendicular gradients of $B$, and to infer the current density from Eq. (1) ignoring these terms. However, as shown below, this is an uncontrolled approximation that can insert arbitrarily large errors into the estimates of $\boldsymbol{J}$ for some sample geometries.

The error made in ignoring the parallel gradients of $\mathbf{B}$ can be obtained by comparison with exact results. Three exact results are available: a long circular wire, a long wire of rectangular cross-section, and an infinite slab. The long circular wire carrying uniform current is an elementary problem. If $\mathrm{J}$ flows along $\mathrm{y}$ and $\mathrm{B}_{\mathrm{z}}$ is measured along a tangent to the surface of the wire in the $x$ direction, the exact solution gives

$$
\frac{\partial B_{z}}{\partial x}=-\frac{2 \pi \mathrm{a}^{2} J}{c} \frac{a^{2}-x^{2}}{\left(a^{2}+x^{2}\right)^{2}}
$$

where $a$ is the radius of the wire. Above the center of the wire $(x=0)$, the exact result is $\partial B_{z} / \partial x=-2 \pi J / c$. Thus estimates of $\mathbf{J}$ based on ignoring the parallel gradients of $\mathbf{B}$ in Eq. (1) are too small by a factor of 2 .

The long wire of rectangular cross-section carrying uniform current density has an exact solution [9] which is algebraically more complicated, but equally useful. If $B_{z}[x]$ is measured along a line tangent to the shorter of the flat sides of the wire. it can be shown that above the center of the wire

$$
\frac{\partial \mathrm{B}_{z}}{\partial \mathrm{x}}=-\frac{2 \pi \mathrm{J}}{\mathrm{c}}\left(1-\frac{\mathrm{w}}{\pi \mathrm{t}}+\cdots\right)
$$

where $w$ and $t$ are the width and thickness of the wire. The error in estimates of $J$ ignoring the parallel gradients of $\mathbf{B}$ in this case is greater than a factor of 2 and depends on the aspect ratio of the wire.

Finally, an infinite slab of uniform current density perpendicular to $z$ can be solved exactly by elementary means. Outside the slab, $B=(2 \pi t / c) \mathbf{J} \times \mathbf{n}$ where $t$ is the thickness of the slab and $\mathbf{n}$ is the outward-drawn normal. $\mathbf{B}$ has opposite direction on opposite sides of the slab but is otherwise uniform outside the slab and has no $z$ component. Thus all of the current is represented by the parallel gradients of $\mathbf{B}$ inside the slab. and estimates of $\mathbf{J}$ based on ignoring them give zero. In this case, the error of such estimates is infinite. In these examples. estimates of the current density based on ignoring the parallel gradients of $\mathbf{B}$ are 
too small by at least a factor of 2 , the error becoming arbitrarily large as the sample thickness shrinks relative to its width.

The errors associated with using the local form of Ampere's law with incomplete information are avoided in principle by using the Blot-Savart law. Here the field outside the sample is properly treated as a non-local integral over all the currents, and the shape of the sample is explicitly taken into account. Whlle exact expressions can be easily written, it is difficult to invert the non-local integral to recover $\mathbf{J}$ from $\mathbf{B}$, and the inversion is, in general, not unique [see, for example, 10]. However, for the case of a long wire of rectangular crosssection, the expansion of the exact solution given by Eq. (3) handles the non-locality and provides a linear relation between the current density and the field gradient for a specific geometry. We take this solution as the basis for our inversion, and use Eq. (3) ignoring the geometrical correction $w / \pi t$. This approach makes two approximations: that the filaments have a small aspect ratio $w / t$ and that their current density is uniform. The first is a controlled approximation, since we know the size of the correction if we know $w$ and $t$. For our imaging geometry, $t$ corresponds to the long and $w$ to the short transverse dimension of the filament, so the aspect ratio correction is small. The approximation of uniform current density can be analyzed as follows. The contribution of a given current element to the field at a distant point falls off as $1 / \mathrm{r}^{2}$. Thus the field at a given point is quite sensitive to the current density nearby, and much less sensitive to currents flowing farther away. Given the large attenuation of $1 / \mathrm{r}^{2}$, even substantial changes in $\mathrm{J}$ at distant points have little effect on the value of the field. The use of Eq. (3) to measure local variations in the current is strongly confirmed by the results reported below: if the local field depended sensitively on distant currents, strong local variations in current density would be unresolvable by this technique. The main effect of using Eq. (3) for local measurements is a smearing of the derived current density. The measured $J$ is an average over a finite region effectively defined by the $1 / \mathrm{r}^{2}$ attenuation of the Biot-Savart law, and the actual variation in $\mathrm{J}$ will be stronger than the measured one.

\section{RESULTS}

The multifilament wire imaged in these experiments is a 37-filament BSCCO 2223 powderin-tube composite $[11,12]$ fabricated by Intermagnetics General Corporation. It had a critical current density of approximately $1.7 \times 10^{4} \mathrm{~A} / \mathrm{cm}^{2}$. in self-field at $77 \mathrm{~K}$, corresponding to a current of 31 A. A. $1.5 \mathrm{~cm}$ long section of this wire was polished to about half its original width, giving final transverse dimensions of $1.37 \times 0.20 \mathrm{~mm}$. Magneto-optical images were taken of the polished surface (defined by the current direction and the short transverse dimension of the wire) while an applied current up to $21 \mathrm{~A}$ was passed through the wire at zero applied field. To avoid heating, the current was applied in pulses of $0.2-0.7$ sec, while the images were recorded with a charge coupled device camera and the voltage developed across the sample was recorded on a storage oscilloscope. Four full filaments were visible on the imaged surface of the wire, as shown in the scanning electron microscope (SEM) image in Fig. Ia. Light gray represents the Ag sheath, dark areas correspond to the superconductor. Also visible in the SEM image are narrow regions of superconductor (labeled "T") which correspond to the tops of filaments buried deeper in the wire, as has been confirmed by additional polishing.

A magnetic field map of the surface produced by magneto-optical imaging is shown in Fig. Ib. The gray scale in the image has been compressed by subtracting a linear background to show all the qualitative features. There is a sequence of light and dark bands running roughly along the current direction, indicating substantial field gradients in the horizontal direction. These gradients are the signature of the current distribution in the filaments, expected on the basis of Eq. (3). The field gradients are shown quantitatively in Fig. 2a. which plots the variation of $\mathrm{B}_{\mathrm{z}}$ along the line indicated at the bottom of Fig. 1. In the region outside the superconducting filaments, the field decays as expected far from a finite current distribution. If the current were carried uniformly in the $\mathrm{Ag}$ sheath, the field would show a constant gradient confined to the region of current flow, as is seen for the data at $100 \mathrm{~K}$ where the BSCCO filaments are normal. At lower temperature, where the BSCCO becomes superconducting. sharp gradients appear over the filaments themselves indicating that 

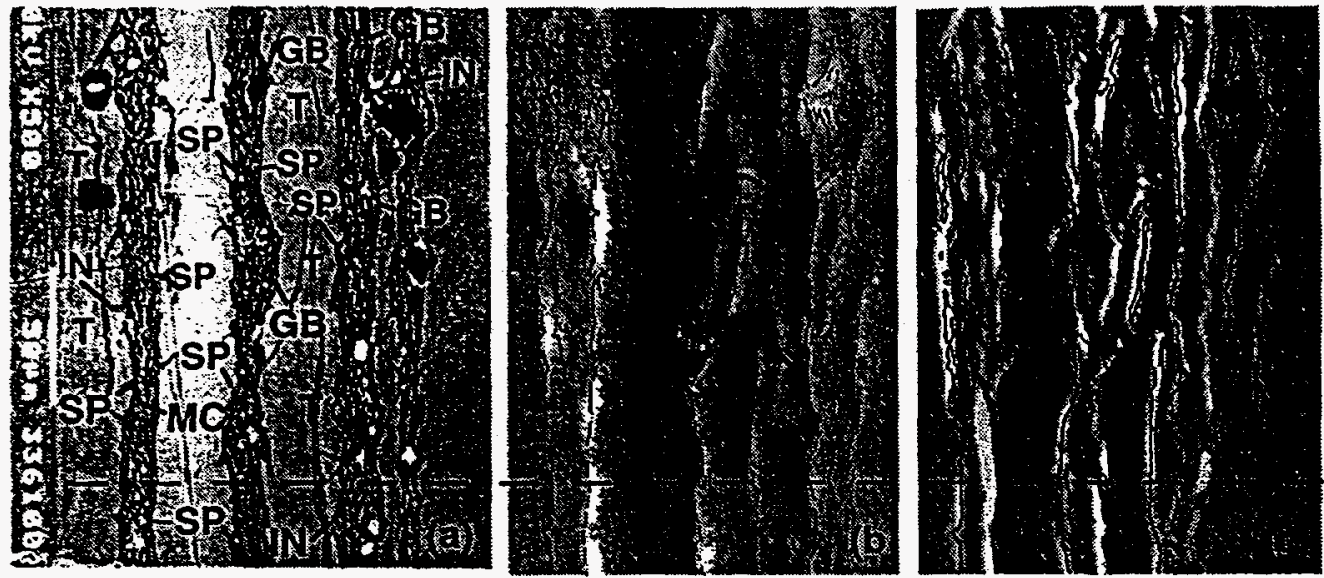

Fig. 1 (a) SEM image of four full filaments visible on the imaged surface. $T=$ tops of filaments partially buried beneath the exposed surface, IN=interconnects between filaments. $\mathrm{SP}=$ second phase particles. $\mathrm{MC}=$ grain colony misaligned with filament direction, GB=low angle deviation in texture. (b) Self-magnetic field pattern with applied current of $21 \mathrm{~A}$. Bright corresponds to positive fields, dark to negative fields. The contrasting light/dark vertical bands are the signature of filaments of current. (c) Current map of the same region. Solid lines indicate the outlines of the filaments. Bright areas correspond to high current density, dark areas to low density.

locally strong currents flow there. These gradients are not constant over the width of the filament, indicating an inhomogeneous current distribution within the filament. The inhomogeneities are most noticeable at the lowest temperature, $30 \mathrm{~K}$ in Fig. 2a. Current sharing in the Ag sheath is indicated by a positive gradient over the outer sheath at $77 \mathrm{~K}$ and $100 \mathrm{~K}$, while at $30 \mathrm{~K}$ the negative gradient over the outer sheath indicates that all the current is being carried by the superconductor.

To convert the field profile in Fig. $2 a$ into a current profile, it is necessary to take the derivative along the horizontal direction, using the prescription of Eq. (3). The current profile is shown in Fig. $2 \mathrm{~b}$, for $30 \mathrm{~K}$ and $77 \mathrm{~K}$. There the current is shown directly, and the
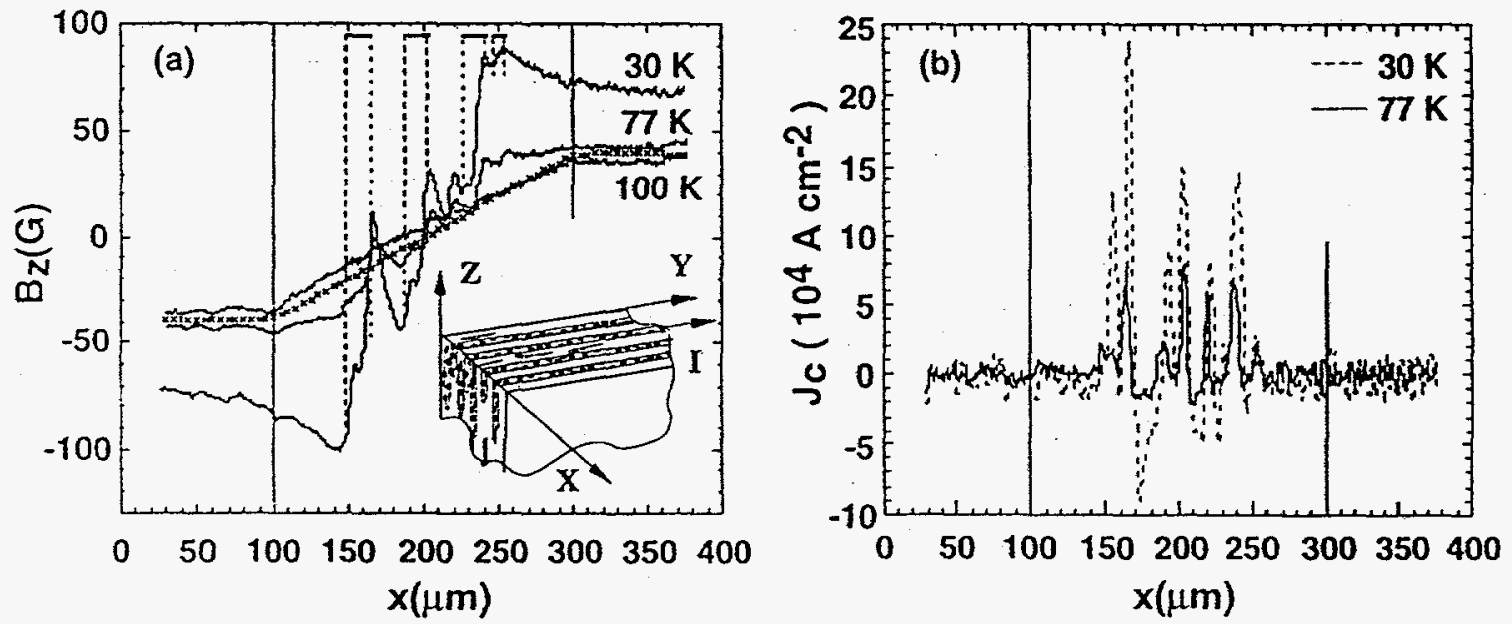

Fig. 2 (a) Magnetic field profile along the dotted line in Fig. I. The solid vertical lines indicate the edges of the Ag sheath, the dotted vertical lines indicate the edges of the superconducting filaments. The inset shows the imaging geometry. (b) Transport current profiles along the same line, obtained by applying Eq: 3 to the field data in (a). 
inhomogeneity within filaments can be easily seen. The uncertainties caused by the unknown aspect ratios of the current carrying grain colonies are less than $25 \%$. (Note that the field gradient in Eq. (3) is evaluated over the center of the current distribution, so It cannot be applied to the regions between filaments. Thus the negative values in Fig. $2 \mathrm{~b}$ in these regions are meaningless and should be ignored.) Regions of remarkably high current density can be found, up to $8 \times 10^{4} \mathrm{~A} / \mathrm{cm}^{2}$ at $77 \mathrm{~K}$. This is much higher than the average current density, $1.7 \times 10^{4} \mathrm{~A} / \mathrm{cm}^{2}$. The current density profiles at $30 \mathrm{~K}$ and $77 \mathrm{~K}$ display the same shape, even though the transport current is below critical in the former and above critical in the latter case. This indicates that the current inhomogeneities within a filament are present even in the fully penetrated critical state, and are not related to the surface flow expected for sub-critical currents.

Full field maps like those in Fig. $1 \mathrm{~b}$ can be converted to current maps by taking the appropriate derivatives via digital image processing. The result is shown in Fig. 1c. The black lines in the current map show the outlines of the filament positions. Dark areas in the current map correspond to zero or low current, light areas to high current. It is apparent that the current density is highly inhomogeneous, varying widely throughout a given filament. Only a fraction of the available superconducting cross-section is effective in carrying current, confirming expectations from slicing [13-15] and microstructural [16-18] studies and earlier magneto-optical studies [4-8]. The apparent termination of local current flow paths in Fig. 1c indicates that the current is diverted beneath the surface. Because of the $1 / r^{2}$ attenuation, such sub-surface paths are not easily seen with our technique.

The availability of high resolution current maps like those of Fig. lc makes possible detailed comparisons of local superconducting performance and the underlying microstructure on the scale appropriate to each. Many interesting details can be seen in the comparison [8]. For example, the current is often seen to follow a few-micron wide strip close to the Ag interface, as has been inferred from earlier mono-fllament slicing studies [13-15] and from magneto-optical imaging in mono- [5,7] and multi-filaments [6]. The interface regions are often seen to contain well-textured, phase-pure microstructure $[17,18]$ which is favorable to high current flow. However. there are important exceptions to this trend, as indicated by the very high current region in the lower part of the left filament, where the current flows in the center of the filament as it crosses from the right edge to the left edge. Examination of the SEM image shows a particularly well-textured microstructure in this region, which guides the current as it winds its way through the superconductor. Similarly, there are long regions of $\mathrm{Ag}$ interface that carry little or no current, such as both edges of the right filament. and the left edge of the second filament from the right. The SEM image shows that there are areas of good microstructure in these regions, but they are often interrupted by second phase particles which obstruct the current flow. Thus, the images show a strong correlation between current density and microstructure on the micron scale, and clearly indicate that the best microstructure and superconducting performance are not necessarily found at the Ag interface.

While the current images demonstrate the spatial correlation of microstructure and performance, they also show that microstructure alone is not enough to guarantee high critical current. There are many regions where good microstructure is seen in the SEM image, but the current density is low. Many such cases occur in the right filament, which carries remarkably little current anywhere along its imaged length. The reason can be found in topology, connectivity, and weak links. Weak links, composed of second phase particles or poor texture, block the current path leading to good material, cutting off access to otherwise high performance regions. The current maps suggest that such connectivity cutoffs are a fairly common occurrence, and a major problem in improving the average critical current of the wire. High resolution current images like the ones presented here illustrate the need and provide the means for making detailed studies of the topology, current path connectivity, and weak links in practical conductors.

Interconnects between filaments play a crucial role in AC loss mechanisms, because they create large area closed loops which support induced eddy currents. In assessing their importance to loss mechanisms, it is necessary to know whether interconnects actively carry current or not. Three interconnects (labeled "IN") can be seen in the SEM image. only one of which, in the lower part of the second filament from the right, carries current. In systems where there are frequent interconnects, current maps provide a new and effective means for exploring their role in contributing to losses. 


\section{SUMMARY}

We have proposed and demonstrated a new analysis technique for converting magnetooptical self-field images to transport current images with spatial resolution in the micron range. The analysis technique is based on an exact solution to the Biot-Savart law for long wires of rectangular cross-section, and does not contain the uncontrolled approximations which limit schemes based on the local form of Ampere's law. We have applied the technique to multi-filament BSCCO 2223 composite superconductors, displaying the transport current distribution with spatial resolution comparable to that of the microstructure. There is a high spatial correlation between superconducting performance and microstructure at the micron level. Good microstructure is often found at the Ag interface, but there are many examples of high current density in the centers of filaments. and of low current density at the Ag interface. The current images show that regions of good microstructure alone do not guarantee good performance, since weak links composed of second phase particles or poorly textured grains may isolate good microstructure from the current path. The role of interconnects in creating large closed loops among filaments can be directly observed; not all interconnects are found to actively carry current. These examples demonstrate that high resolution transport current maps offer a potentially powerful means for understanding and improving the performance of superconducting material.

This work was supported by the U. S. Department of Energy under contract \#W-31-109ENG-38 through the Offices of Basic Energy Sciences- Materials Sciences (GWC. UW) and Energy Efficiency and Renewable Energy in a program to develop electric power technology (UB, WZ), by the U. S. National Science Foundation-Science and Technology Center for Superconductivity under contract \#DMR-91-20000 (DOG), by the New Independent StatesIndustrial Partnership Program (VKV), and by the International Science Foundation under grants \#RF1000 and \#RF1300 (VKV, VIN).

\section{REFERENCES}

1. Dorosinskii LA, Indenbom MV, Nikitenko VI, Ossip'yan AA, Polyanskii AA, VlaskoVlasov VK (1992) Physica C 203: 149-156

2. Vlasko-Vlasov VK, Indenbom MV, Polyanskii AA (1993) In: Shekhtman V (ed) The Real Structure of High Tc Superconductors. Springer. Berlin p 111

3. Welp U, Gardiner T, Gunter DO, Fendrich JA. Crabtree GW, Vlasko-Vlasov VK, Nikitenko VI (1994) Physica C 235-240: 241-244

4. Vlasko-Vlasov VK, Goncharov VN, Nikitenko VI, Polyanskii AA. Voloshin IF. Fisher LM, Aleshina NM, Poluschenko OA (1994) Physica C 222: 367-374

5. Pashitskii AE, Polyanskii AA, Gurevich A, Parrell JA, Larbalestier DC (1995) Physica C 246: $133-144$

6. Welp U, Gunter DO, Crabtree GW, Luo JS, Maroni VA, Carter WL, Vlasko-Vlasov VK, Nikitenko VI (1995) Appl Phys Lett 66: 1270-1272

7. Pashitskii AE, Polyanskii AA. Gurevich A, Parrell JA, Larbalestier DC (1995) Appl Phys Lett (In Press)

8. Welp U. Gunter DO, Crabtree GW. Zhong W, Balachandran U, Haldar P, Sokolowski RS, Vlasko-Vlasov VK, Nikitenko VI (1995) Nature 376: 44-46

9. Binns KJ, Lawrenson PJ (1963) Analysis and Computation of Electric and Magnetic Field Problems. Pergamon. Oxford

10. Brandt H (1992) Phys Rev B 46: 8628-8631

11. Sandhage K, Carter WL, Riley GN (1991) J. Minerals Metals \& Materials Soc 43: 21-25

12. Balachandran U, lyer AN, Huang JY, Jammy R, Haldar P, Hoehn JG, Galinski G, Motowidlo LR (1994) J. Minerals Metals \& Materials Soc 46: 23-25

13. Larbalestier DC, Cai XY, Feng Y, Edelman H, Umezawa A, Riley GN, Carter WL (1994) Physica C 221: 299-303

14. Grasso G. Hersel B, Jeremie A, Flukiger R (1995) Physica C 241: 45-52

15. Lelovic M, Krishnaraj P, Eror NG. Balachandran U (1995) Physica C 242: 246-250

16. Yamada Y. Satou M. Murase S (1993) In: Bando Y, Yameuchi H (eds) Proc of $5^{\text {th }}$ Int'1 Symposium on Superconductivity. Springer. Tokyo, p 717

17. Liu HK. Wang RK, Dou SX (1994) Physica C 229: 39-46

18. Merchant N, Luo JS. Maroni VA. Riley GN, Carter WL (1994) Appl Phys Lett 65: 1039-1041 